\title{
Arterial ketone index in assessing liver function and its detoxicative capability after ischemia-reperfusion injury ${ }^{*}$
}

\author{
Artur Caban ${ }^{1}$, Ryszard Wiaderkiewicz ${ }^{2 凶}$, Marcin Kamiński ${ }^{2}$, Grzegorz Oczkowicz ${ }^{1}$ \\ and Jacek Ziaja ${ }^{1}$
}

${ }^{1}$ Department of General and Transplantation Surgery, and ${ }^{2}$ Department of Histology and Embryology, Silesian Medical University, Katowice, Poland

Received: 23 August, 2000; revised: 24 October, 2000; accepted: 15 November, 2000

Key words: arterial ketone index, ischemia, reperfusion, cytochrome P450

\begin{abstract}
Arterial ketone index (AKBR) which is the ratio of acetoacetic acid to 3-hydroxybutyric acid in the arterial blood, is believed to reflect the mitochondrial reduction potential of hepatocytes and general energy state of the liver. In the presented paper we challenged this hypothesis by analysing the correlation between AKBR and the results of typical liver blood tests (AspAT, AlAT, LDH, CRP) and biotransforming potential of the liver (cytochromes $\mathrm{P}_{450}, b_{5}$ and their corresponding NADPH and NADH reductases) in the model of ischemia-reperfusion injury of rat liver. The results were compared with histochemical analysis of distribution and activity of SDH, LDH and G-6-Pase, the key marker enzymes of the liver. We have shown that, except in the case of acute phase protein (CRP), a decrease in AKBR correlated well with the increase of the level of indicator enzymes in serum. Histochemical analysis also confirmed that AKBR correlates with the degree of damage to hepatocytes during early stage of reperfusion after 60 min of liver ischemia. In the Spearman test, AKBR was significantly correlated with the changes in cytochrome P450 content and its NADPH reductase activity which indicates a high sensitivity of this test. We conclude that the decrease of AKBR value reflects the impairment of basic energy pathways and detoxicative capability of the liver.
\end{abstract}

\footnotetext{
This work was supported by the State Committee for Scientific Research (KBN, Poland) grant 4 P05D 07015

${ }^{凶}$ Corresponding author: R. Wiaderkiewicz, Department of Histology and Embryology, Silesian Medical University, Medyków 18, 40-762 Katowice, Poland; tel/fax. (48 32) 252 6574; e-mail: histem2@infomed.slam.katowice.pl
}

Abbreviations: AKBR, arterial ketone index; AlAT, alanine aminotransferase; AspAT, aspartate aminotransferase; CRP, C-reactive protein; G-6-Pase; glucose-6-phosphatase; IR, ischemia/reperfusion; $\mathrm{LDH}$, lactate dehydrogenase; SER, smooth endoplasmic reticulum; SDH, succinate dehydrogenase. 
Assessment of liver functioning early after a surgery is a crucial factor in determination of future prognosis and treatment. This is especially important in those cases when temporary ischemia of the organ followed by its reperfusion takes place. This occurs during liver transplantation, extensive resections by the Pringle's manoeuvre, post-traumatic shock, haemorrhagic shock, and cardiac shock. Damages developed during ischemia are intensified to a significant degree by the processes occurring after restoration of blood circulation and are generally described as the ischemia-reperfusion (IR) injury. Specificity of the liver response to IR is a result of its high oxygen demand, unique arterial-portal vascularisation and extensive metabolic and biotransformation functions of this organ. Furthermore, it is a result of a complex anatomic and functional interdependence of hepatocytes and non-parenchymal cells of liver sinuses such as endothelium cells, Kupffer cells, stellate (Ito) cells, and neutrophils.

Standard liver tests reflect only to a small degree the processes occurring during IR and cannot assess the regenerative and detoxicative capabilities of the damaged organ. On the other hand, more precise functional, histochemical, and enzymatic tests have not met general clinical approval as they are too complex, time-consuming and expensive. The arterial ketone index (AKBR), which is the ratio of acetoacetic acid to 3-hydroxybutyric acid, was introduced in clinical practice in 1981. It reflects to a great extent the mitochondrial reduction potential of hepatocytes and energy state of the liver [1]. AKBR was found to be useful as an independent prognostic factor in liver transplantation, haemorrhagic shock, septic shock, after application of the Pringle's manoeuvre during liver resection, and after cardiosurgery [2, 3]. Long-term prognosis in those cases is far better if AKBR is higher, as proven empirically and clinically [4-6]. However, the significance of the ketone index in liver damage assessment at the early stage after extensive organ resection has been questioned [7, 8].

The goal of this work was to evaluate the usefulness of AKBR determination at the early stage of reperfusion after $60 \mathrm{~min}$ of ischemia. The obtained values of the ketone index were compared with the results of standard liver tests for: alanine aminotransferase (AlAT), aspartate aminotransferase (AspAT), lactate dehydrogenase (LDH) and C-reacting protein (CRP). Histochemical reactions to succinate dehydrogenase (SDH), LDH and glucose-6phosphatase (G-6-Pase) were analysed by microscopy to evaluate degree of the damage to the liver. Finally, we correlated AKBR with the content and activities of cytochrome $\mathrm{P} 450$, cytochrome $b_{5}$ and their corresponding reductases which are crucial components of the biotransforming machinery of the liver.

\section{MATERIALS AND METHODS}

Three months old inbred male rats (Spraque-Dawley) which gained body mass of 230-250 g were used in the experiments. The animals were kept separately in plastic cages at ambient temperature of $23^{\circ} \mathrm{C}$, relative humidity of $70 \%$, constant day-night cycle 12/12 $\mathrm{h}$ and free access to food and water. To standardise the conditions all the operations were carried out always at the same time between $8.00 \mathrm{am}$ and $11.00 \mathrm{am}$. For $12 \mathrm{~h}$ before surgery the animals were provided with water only. After standard intraperitoneal anaesthesia (ketamine $10 \mathrm{mg} / \mathrm{kg}$ of body mass plus droperidol $0.25 \mathrm{mg} / \mathrm{kg}$ of body mass) the peritoneal cavity was opened by median incision. The model of partial liver ischemia with decompression of visceral circulation was made according to Hasselgren et al. [9]. After the vessels of hilus of the liver had been dissected free, the vessels leading to the middle and left liver lobe (which constitute $70 \%$ of the liver mass) were clamped with clips Yasargil ${ }^{\circledR}$ Aesculap (Germany). At the same time the flow through the right and spigelian lobe was 
maintained to avoid deadly visceral stasis, bacterial translocation through intestine walls and secondary endotoxaemia. Immediately after the liver circulation was restored, spigelian and right liver lobes were resected to eliminate the influence of their metabolism on the outcome of the experiment. The abdominal cavity was then closed with a continuous suture and the animals were placed separately in plastic cages.

The following groups, each containing 10 individuals, were studied:

- I, randomly chosen, sham operated control animals;

- II, animals subjected to $60 \mathrm{~min}$ of ischemia and $30 \mathrm{~min}$ of reperfusion;

- III, animals subjected to $60 \mathrm{~min}$ of ischemia and $72 \mathrm{~h}$ of reperfusion.

After reperfusion the animals were killed by decapitation and exsanguinated. The serum concentration of AspAT, AlAT, LDH and CRP were determined in blood samples (autoanalyzer Mira-Cobas ${ }^{\circledR}$ produced by Roche). To calculate the ketone index the acetoacetic and beta-hydroxybutyric acids were determined in arterial blood according to Wiliamson et al. (Ketorex ${ }^{\mathrm{TM}}$ Sanwa, Nagoya, Japan) [10].

Pieces of the liver taken from the left liver lobe were immediately frozen in compressed carbon dioxide and placed in cryostat CRYO-CUT (American Optical Corporation) at a temperature of $-15^{\circ} \mathrm{C}$. Specimens were cut into serial pieces $7 \mu \mathrm{m}$ thick and placed on cold gelatine coated slides. In such preparations the activities of the following enzymes were checked histochemically: SDH (EC 1.3.99.1) and LDH (EC 1.1.1.27) by Nachlas method according to Pearse [11] using Tetra Nitro BT sodium salt produced by Sigma, and G-6-Pase (EC 3.1.3.9) by Wachstein-Meisel method according to Pearse [11] using glucose-6-phosphate sodium salt produced by Koch-Light. The control of the reaction specificity was always carried out at the same time according to the good laboratory practice. Photographic documentation was made using microscope Reihert-Polyvar and black and white FOTON films (sensitivity of 10 DIN).

Immediately after dissection, the part of the liver was rinsed in ice cold $0.14 \mathrm{M} \mathrm{NaCl}$, cut into small pieces and homogenized at 400 r.p.m. in $0.25 \mathrm{M}$ sucrose in $10 \mathrm{mM}$ Tris/ $\mathrm{HCl}$ buffer, $\mathrm{pH}$ 7.5, in Potter-Elvehjem homogenizer (Braun Melsungen AG) equipped with Teflon piston. The obtained homogenate was centrifuged for $20 \mathrm{~min}$ at $1000 \times \boldsymbol{g}$ and the postmitochondrial supernatant was ultracentrifuged (Beckman L5-75) at $105000 \times \mathbf{g}$ for $90 \mathrm{~min}$. The pellet containing the microsomal fraction was suspended in $10 \mathrm{mM}$ Tris/ $\mathrm{HCl}$ buffer containing $0.025 \mathrm{M}$ sucrose and $2 \%$ glycerol and subjected to following examinations: cytochrome P450 and cytochrome $b_{5}$ content according to Estabrook \& Werringloer [12], the activity of NADPH-cytochrome $\mathrm{P} 450$ reductase and $\mathrm{NADH}-$ cytochrome $b_{5}$ reductase according to Hodges \& Leonard [13].

Statistical analysis was carried out using commercial software STATISTICA ${ }^{\circledR}$ V.5.0 for Windows. The Shapiro-Wilk test (checking the fit with Gaussian distribution) and Behrens-Fisher and Van der Waerden test (comparing two mean distributions) were used. Correlation was determined using Spearman test. Values of $P<0.05$ were considered statistically significant.

\section{RESULTS}

\section{Biochemical tests}

All the animals tested survived the treatment and the planned reperfusion time. The results of basic biochemical tests are presented in Table 1. As compared with groups I and III, in group II (60-min ischemia, $30 \mathrm{~min}$ of reperfusion) AspAT and $\mathrm{LDH}$ values were statistically significantly higher. However, the level of AlAT and CRP was increased non-significantly. In this group the ketone index was significantly decreased to on average 0.112. 
After $72 \mathrm{~h}$ of reperfusion (group III) normalization of all the above values was observed. cytochrome $b_{5}$ activity revealed results similar to those for cytochrome P450 - a signifi-

Table 1. Biochemical analysis of blood.

Group I, control; group II, $60 \mathrm{~min}$ ischemia/30 min reperfusion; group III, $60 \mathrm{~min}$ ischemia/72 h reperfusion. S.D., standard deviation.

\begin{tabular}{llccccc}
\hline & Group & Mean & Mediana & Minimum & Maximum & S.D. \\
\hline $\begin{array}{l}\text { AlAT } \\
\text { (U/L) }\end{array}$ & I & 55.2 & 56 & 44 & 63 & 7.12 \\
& II & 541.2 & 380 & 300 & 1275 & 411.958 \\
& III & 91.8 & 80 & 52 & 165 & 45.752 \\
AspAT & I & 180 & 182 & 160 & 202 & 16 \\
(U/L) & II & 4132.4 & 3570 & 2116 & 7398 & 1975.487 \\
& III & 257.2 & 192 & 126 & 602 & 194.682 \\
LDH & I & 251.2 & 250 & 194 & 310 & 45.861 \\
(U/L) & II & 7647.6 & 8110 & 1514 & 12540 & 3989.63 \\
& III & 907.2 & 780 & 293 & 1360 & 445.875 \\
CRP & I & 15.2 & 15 & 14 & 18 & 1.643 \\
(mg/L) & II & 22.8 & 22 & 14 & 30 & 6.14 \\
& III & 15.4 & 14 & 9 & 23 & 6.348 \\
AKBR & I & 0.918 & 0.930 & 0.720 & 1.03 & 0.123 \\
& II & 0.112 & 0.100 & 0.050 & 0.17 & 0.045 \\
& III & 0.418 & 0.160 & 0.070 & 1.03 & 0.433 \\
\hline
\end{tabular}

In the case of AlAT and CRP the results did not differ significantly from those for the control group. AKBR reached a mean value of 0.418 but it was still significantly lower as compared with the control group. However, there was no significant difference in comparison with group II.

The applied trauma (IR) caused a significant decrease in the activities of enzymes involved in the first phase of biotransformation in the liver (Table 2). Cytochrome P450 level decreased significantly in group II, however, after $72 \mathrm{~h}$ of reperfusion it increased considerably (group III). The activity of NADPH reductase was reduced in both groups after reperfusion $(P<0.05)$. Statistical analysis of cant decrease in group II and increase in group III, almost to the level of the control group. Similarly, after $72 \mathrm{~h}$ of reperfusion, the activity of NADH reductase surpassed slightly the values for the control group.

\section{Histochemical tests}

Succinate dehydrogenase

A strong granular-diffusive reaction was observed in all the hepatocytes of the liver acini in group I. A strong reaction evident in parenchymal cells of the acinar zone 1 decreased gradually toward zones 2 and 3 (Fig. 1a). In groups II and III a gradual decrease in the in- 
tensity of the enzymatic reaction was observed, beginning in zone 1 and then visible in the remaining zones (Fig. $1 \mathrm{~b}$ and c). After 30 min of reperfusion, the reaction was mainly diffusive and a part of the parenchymal cells showed no reaction at all (Fig. 1b). Within all acinar zones single hepatocytes showing a
Glucose-6-phosphatase

In group I the midgranular-diffusive reaction was localized in the cytoplasm of the hepatocytes in the whole liver, being the most intense in zone 1 (Fig. 2a). With elongation of the reperfusion time (groups II and III) the
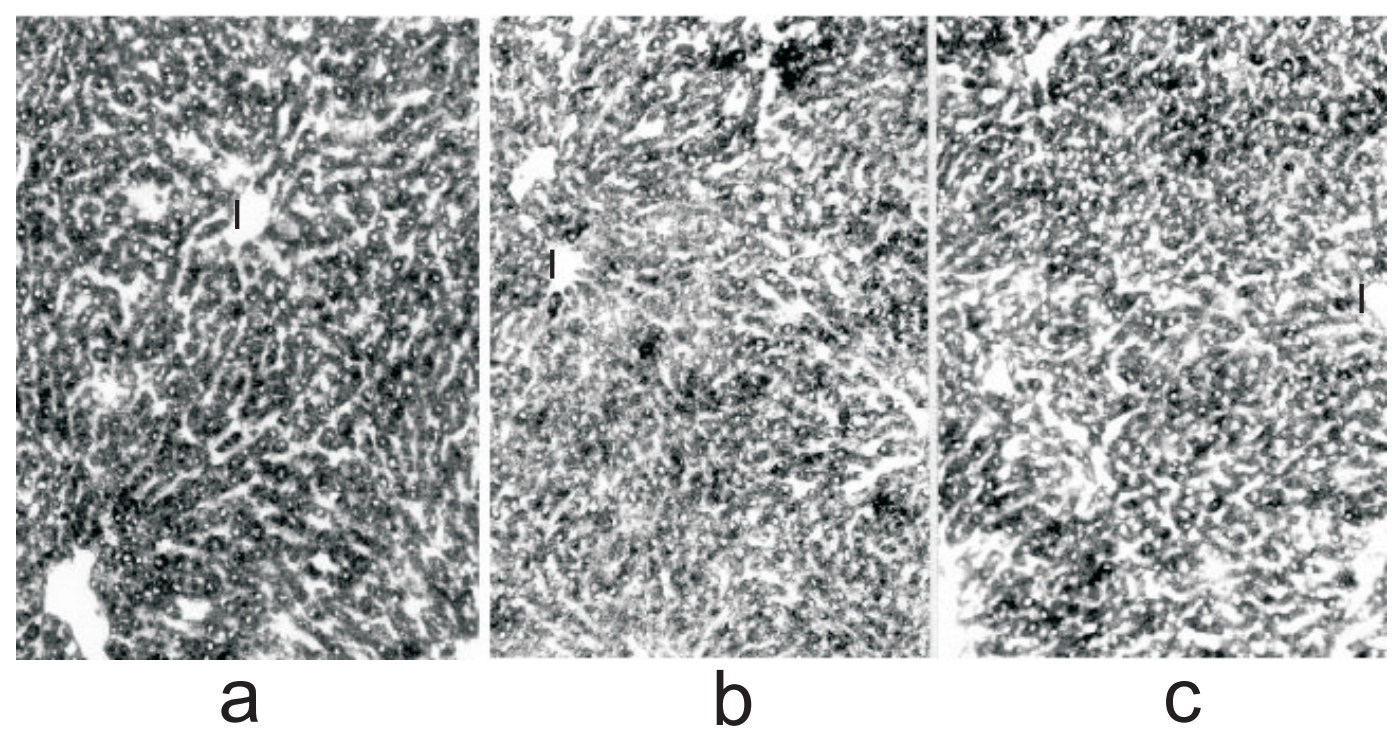

Figure 1. Histochemical reaction for the presence of succinic dehydrogenase.

Asterisk indicates central vein of liver acinus with surrounding hepatocytes forming zone 3. a, Control (group I); b, $60 \mathrm{~min}$ ischemia/30 min reperfusion (group II); c, $60 \mathrm{~min}$ ischemia/72 h reperfusion (group III).

considerably stronger reaction were observed. In group III foci of not reacting hepatocytes were surrounded by hepatocytes exhibiting a strong diffusive reaction (Fig. 1c).

Lactate dehydrogenase (not shown)

In group I (control) the diffusive reaction in the cytoplasm of the hepatocytes was observed. The reaction was intensive in zone 1 , decreasing gradually toward zones 2 and 3 . In group II (30 min of reperfusion) a weaker reaction was seen in all acinar zones. However, some hepatocytes still reacted quite strongly (but less than in group I). After $72 \mathrm{~h}$ of reperfusion (group III) oval areas of parenchyma showing a negative reaction were noticed. In the necrotic areas (zones 3 and 2) a weaker diffusive reaction was observed in single cells. granular-diffusive reaction diminished significantly and become diffusive in most of the parenchymal cells (Fig. 2b and c). After $72 \mathrm{~h}$ of reperfusion the foci of hepatocytes not exhibiting a positive reaction were seen in zones 2 and 3 (Fig. 2c).

\section{Correlation analysis}

Correlation between the ketone index and other biochemical markers are presented in Table 3. In our experiments a decrease of the ketone index correlated with an increase of the levels of indicator enzymes in serum. No similar relationship was observed as regards the acute phase protein (CRP). A statistically significant relationship occurred between AKBR and cytochrome P450 content and NADPH reductase activity (Fig. 3). Changes in the cytochrome $b_{5}$ content and NADH 


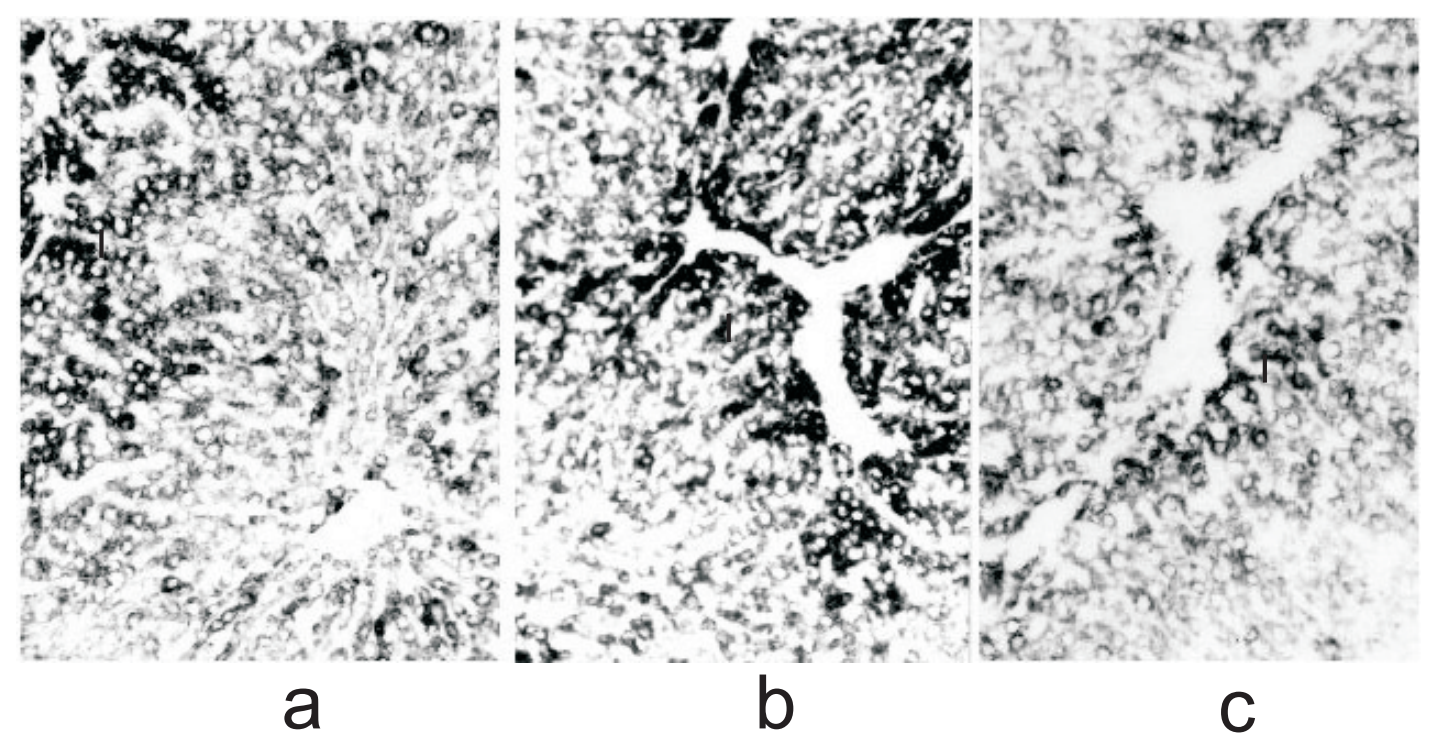

Figure 2. Histochemical reaction for the presence of glucose-6-phosphatase.

Asterisk indicates periportal area (zone 1). a, Control (group I); b, $60 \mathrm{~min}$ ischemia/30 min reperfusion (group II); c, $60 \mathrm{~min}$ ischemia/72 h reperfusion (group III).

reductase activity did not show any correlation with the changes in AKBR.

\section{DISCUSSION}

The use of Hasselgren model allowed to apply ischemia of $70 \%$ of liver (two lobes) for 60 min. During surgery, no traits of venostasis within the abdominal cavity organs or hemodynamic instability were observed. All rats survived the treatment and the reperfusion time. This allowed to compare structural and functional changes in the liver observed after different times of reperfusion. The model applied allowed to eliminate venostasis and, thus, the potential influence of bacterial dislocation on stimulation of Kupffer cells, which could obscure the effects of ischemia-reperfusion trauma.

Ischemia lasting $60 \mathrm{~min}$, even though it was well tolerated by rats, resulted in meaningful changes in the analysed histochemical reactions and biochemical tests. In the case of $\mathrm{SDH}$ and LDH an increase in the amount of the reaction product within all acinar zones is striking, although the granulate-diffusive character of the SDH reaction is sized and

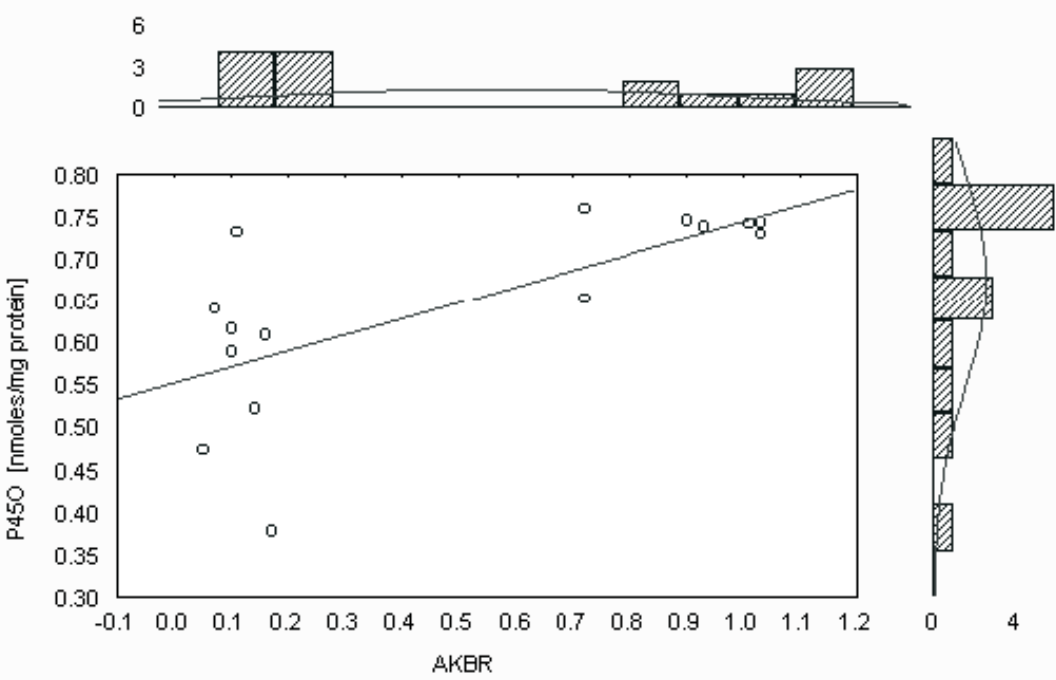

Figure 3. Analysis of correlation between AKBR and cytochrome P450 (Spearman test).

$\mathrm{R}=0.649958, \quad \mathrm{P}=0.008717$ 
there is a difference between distribution of the two enzymes in individual zones. SDH activity correlates well with the state and quantity of mitochondria in hepatocytes. In the intact animals the amount of mitochondria reaches 2000 /cell and is the highest in zone 1 , decreasing progressively towards zone 3 . $\mathrm{SDH}$ forms the enzymatic complex II of the electron transport chain, and its activity re- brane by phospholipase $\mathrm{A}_{2}$ (activated by high calcium level in mitochondria). There is an unquestionable correlation between the reaction of SDH and increment of AspAT level in serum of the animals in group II. AspAT is an enzyme less specific for the liver but still more sensitive than AlAT. AspAT originates in 80\% from damaged mitochondria and AlAT, similarly as LDH, is mainly released into blood

Table 2. Content of cytochrome $\mathbf{P} 450$ (nmoles $/ \mathrm{mg}$ protein), cytochrome $b_{5}$ (nmoles/mg protein) and activities of their corresponding reductases (mmoles/min per mg protein) in microsomal fraction of the liver.

Group I, control; group II, $60 \mathrm{~min}$ ischemia/30 min reperfusion; group III, $60 \mathrm{~min}$ ischemia/72 h reperfusion. S.D., standard deviation.

\begin{tabular}{lllcccc}
\hline & Group & Mean & Mediana & Minimum & Maximum & S.D. \\
\hline \multirow{2}{*}{ Cyt P450 } & I & 0.746 & 0.744 & 0.738 & 0.76 & 0.009 \\
& II & 0.516 & 0.523 & 0.379 & 0.62 & 0.095 \\
& III & 0.673 & 0.652 & 0.609 & 0.73 & 0.055 \\
\hline \multirow{2}{*}{ NADPH-cyt P450 0} & I & 0.137 & 0.137 & 0.126 & 0.14 & 0.006 \\
reductase & II & 0.079 & 0.081 & 0.058 & 0.10 & 0.014 \\
& III & 0.110 & 0.110 & 0.107 & 0.11 & 0.003 \\
Cyt $b_{5}$ & I & 0.585 & 0.562 & 0.544 & 0.67 & 0.052 \\
& II & 0.392 & 0.402 & 0.355 & 0.42 & 0.025 \\
& III & 0.510 & 0.511 & 0.433 & 0.60 & 0.060 \\
NAD-cyt $b_{5}$ & I & 0.781 & 0.781 & 0.733 & 0.81 & 0.031 \\
reductase & II & 0.635 & 0.624 & 0.580 & 0.75 & 0.065 \\
& III & 0.823 & 0.801 & 0.757 & 0.96 & 0.081 \\
\hline
\end{tabular}

flects the state of oxidation-reduction systems in the inner mitochondrial membrane. These systems are of crucial importance for cell energy metabolism. The change of the reaction character into granular-diffusive and diffusive in group II proves that only a part of mitochondria were damaged and that they were very active in zones 2 and 3 . Literature data point to high resistance of these organelles to ischemia : lack of structural changes, and ability to oxidative phosphorylation during short periods of ischemia. Only over 90-min ischemia leads to a damage to the mitochondrial respiratory chain, which occurs probably as a result of disordering of the inner mem- from cytosol. The results presented by Shimizu et al. [14] showed that, during reoxygenation of isolated liver, the increase in AspAT level affected mainly the mitochondrial fraction. The use of cycloserine, which decreases the permeability of mitochondrial membrane to calcium ions, caused a lower release of mitochondrial AspAT. Interestingly, the increase in activity of this enzyme was seen during reoxygenation but not during ischemia. Similarly Gonzales-Flecha et al. [15] did not observe increased concentration of AspAT during the ischemia period but only during reperfusion. In our tests the concentration of the two aminotransferases reached 
the highest levels after 30 min of reperfusion. After $72 \mathrm{~h}$ of reperfusion (group III) the concentration of AspAT dropped almost to the control level, however, there was still an increase in LDH level in serum. A histochemically significant increment of the diffusive reaction of $\mathrm{LDH}$ was observed after 30 min of reperfusion, in group II, especially in acinar zones 2 and 3 . An experiment by Lemasters et al. [16] can provide one of the explanations of this fact. After $15 \mathrm{~min}$ of ischemia he observed "blisters", located mainly in zone 3 , which consisted of cytosol work. Glucose-6-phosphatase localized in the form of small granules on the outer surface of endoplasmic membranes is a histochemical marker of smooth endoplasmic reticulum (SER). The G-6-Pase activity in the control group was especially intensive in the hepatocytes located in acinar zone 1 , containing a high amount of SER. This typical topography of G-6-Pase was not observed in group II where this activity was generally low and only few hepatocytes still showed the diffusive reaction. The low G-6-Pase level was undoubtedly connected with increased degradation of

Table 3. Analysis of correlation between AKBR and other biochemical markers in blood (AspAT, AlAT, LDH, CRP) and microsomal fraction (cyt P450, cyt $b_{5}$, NADPH-cyt P450 reductase, NADH-cyt $b_{5}$ reductase)

\begin{tabular}{|c|c|c|c|c|}
\hline & Correlation between AKBR and: & R Spearman & $\mathrm{T}(\mathrm{N}-2)$ & $P$ \\
\hline \multirow{4}{*}{$\begin{array}{l}\text { : } \\
\frac{0}{\infty}\end{array}$} & AspAT & -0.538946 & -2.30690 & 0.038167 \\
\hline & AlAT & -0.796780 & -4.75422 & 0.000377 \\
\hline & LDH & -0.682187 & -3.36397 & 0.005082 \\
\hline & CRP & -0.217202 & -0.80229 & 0.436809 \\
\hline \multirow{4}{*}{ 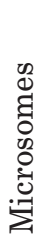 } & Cytochrome P450 & 0.649958 & 3.08361 & 0.008717 \\
\hline & NADPH-cyt P450 reductase & 0.558641 & 2.42849 & 0.030417 \\
\hline & Cytochrome $b_{5}$ & 0.393914 & 1.54521 & 0.146284 \\
\hline & $\mathrm{NADH}$-cyt $b_{5}$ reductase & 0.363475 & 1.40674 & 0.182958 \\
\hline
\end{tabular}

without cellular organelles. Further analysis, including chromatography, revealed that "blisters" were not absorbed but their content rich in LDH entered the blood circulation system. This phenomenon could explain the early increase in LDH level in serum, before damage to the hepatocytes, causing the release of AspAT from mitochondria and AlAT from cytozol, did occur.

Anoxia, compensatory glycogenolysis, and lack of ATP evoke a disturbance in cellular homeostasis [17]. This leads to $\mathrm{pH}$ lowering, inhibition of ionic pumps activity, increase of intracellular content of calcium and decrease of potassium ions which in turn affects the structure and function of essential cellular organelles including the endoplasmatic net- glycogen, intensified during the ischemia period, and lack of its restoration in anaerobic conditions. After $72 \mathrm{~h}$ of reperfusion the foci of necrosis in zone 1 and especially in zone 3 could be seen. Such changes were visible in all histochemical reactions. Early, relatively small damage to mitochondria, distinctly increased with the time of reperfusion. After 30 min of reperfusion most of the hepatocytes attempted to recover their functions. At $72 \mathrm{~h}$ after blood circulation was restored diversity was even greater. The LDH activity in response to reperfusion was increased in group II, followed by slow and gradual decrease in group III. After $72 \mathrm{~h}$ of reperfusion areas of necrosis and a weak diffusive reaction was found in single cells. Undoubtedly it was due 
to the earlier described $\mathrm{LDH}$ release and relatively late availability of substrates for the anaerobic energy pathways after blood flow restoration.

Histochemical analysis corresponded well with AKBR. Considerable damage to hepatocytes observed in group II caused a statistically significant decrease of the ketone index to, an average, 0.112. Partial restoration of energy pathways in cells was accompanied by a threefold increase of AKBR although, its value was still significantly lower than in the control group. A correlation between the ketone index and other marker enzymes was also found.

One of the most important functions of hepatocytes is their participation in metabilism of endogenous substrates and detoxification of xenobiotics. Key components of this system are located mostly in the membranes of smooth endoplasmic reticulum and consist of cytochrome P450, cytochrome $b_{5}$, $\mathrm{NADPH}$-cyt $\mathrm{P} 450$ reductase and $\mathrm{NADH}$-cyt $b_{5}$ reductase. The decrease of ATP and oxygen level observed during the ischemia period, leads to a decrease in activities of the above mentioned enzymes. Already after 30 min of reperfusion a considerable decrease of the level of all of the above mentioned enzymes was observed, which is consistent with literature reports. Izuishi et al. [18] observed a $77 \%$ decrease of cytochrome P450 content after $60 \mathrm{~min}$ of ischemia and $24 \mathrm{~h}$ of reperfusion. Similarly, Chien \& Farber [19] reported that the greatest changes occurred during reperfusion. Depression of cytochrome $\mathrm{P} 450$ content and its transformation to non-active cytochrome $\mathrm{P} 420$ could be a result of its high sensitivity to IR due to changes in phospholipid environment of smooth endoplasmic reticulum induced by phospholipase $\mathrm{A}_{2}$ during ischemia and by peroxidase during the reperfusion phase. It has been shown that cytochrome P450 is not just one of the targets of IR injury but may, as well, be a part of its destructive machinery by generating free oxygen radicals and subsequent lipid peroxidation. The level of cyto- chromes $\mathrm{P} 450$ and $b_{5}$ returns to the control values only after 2-3 weeks of reperfusion. The corresponding reductases behave in a similar way. Their activity during reperfusion is additionally decreased by increased oxidation of NADH and NADPH after restoration of blood circulation. After $72 \mathrm{~h}$, despite the increase of cytochrome $\mathrm{P} 450$ content, the activity of its reductase was statistically significantly lower than in the control group. The results of Spearman test indicated that AKBR was significantly correlated with the changes of cytochrome P450 content and NADPH-cyt $\mathrm{P} 450$ reductase activity. However, no similar correlation with cytochrome $b_{5}$ and its reductase was observed.

We conclude that the arterial ketone index correlates significantly with the degree of damage to the hepatocytes during early stage of reperfusion after $60 \mathrm{~min}$ of liver ischemia. The observed decrease of AKBR value reflects the impairment of basic energy pathways and detoxicative liver capability. The positive correlation between cytochrome $\mathrm{P} 450$ content and AKBR indicates that this test is highly sensitive.

\section{R E F E R E N C E S}

1. Terasaki, M., Patel, N.H., Helton, W.S., Coldwell, D.M., Althaus, S.J., Morimoto, T., Yamaoka, Y., Ozawa, K. \& Nelson, J.A. (1998) Effects of transjugular intrahepatic portosystemic shunts on hepatic metabolic function determined with serial monitoring of arterial ketone bodies. J. Vasc. Interv. Radiol. 9, 129-135.

2. Hanazaki, K., Wakabayashi, M., Sodeyama, H., Makiuchi, A., Igarashi, J., Yokoyama, S., Sode, Y., Kawamura, N. \& Miyazaki, T. (1998) Arterial ketone body ratio does not correlate with ischemic changes during major hepatectomy. Hepatogastroenterology 45, 145-149.

3. Saibara, T., Maeda, T., Onishi, S. \& Yamamoto, Y. (1994) The arterial blood ketone body 
ratio as a possible marker of multi-organ failure in patients with alcoholic hepatitis. Liver 14, 85-89.

4. Nakatani, T., Spolter, L. \& Kobayashi, K. (1995) Arterial ketone body ratio as a parameter of hepatic mitochondrial redox state during and after hemorrhagic shock. World J. Surg. 19, 592-596.

5. Tokuka, A., Kitai, T., Tanaka, A., Yanabu, N., Sato, B., Mori, S., Inomoto, T., Shinohara, H., Yamaoka, Y., Uemoto, S., Tanaka, K., Someda, H., Fujimoto, M. \& Moriyasu, F. (1996) Analysis of factors affecting the graft oxygenation state in living related liver transplantation. Hepatogastroenterology 43, 12031211.

6. Yamaoka, Y., Washida, M., Manaka, D., Gubernatis, G., Ringe, B., Ozaki, N., Yamaguchi, T., Takada, Y., Ollerich, M. \& Ozawa, K. (1993) Arterial ketone body ratio as a predictor of donor liver viability in human liver transplantation. Transplantation 55, 92-95.

7. Hanazaki, K., Wakabayashi, M., Sodeyama, H., Sode, Y., Kawamura, N., Miyazaki, T. \& Ohtsuka, M. (1997) Arterial ketone body ratio during hepatectomy. Hepatogastroenterology 44, 1438-1444.

8. Kameoka, N., Chijiiwa, K., Kozaki, N., Makino, I., Naito, T. \& Tanaka, M. (1996) Hepatic adenine nucleotides and DNA synthesis during the regenerative and atrophic process of the liver lobes after selective portal vein ligation. Eur. Surg. Res. 28, 212-221.

9. Hasselgren, P.O., Jennische, E., Fornander, J. \& Hellman, A. (1982) No effect of ATP- $\mathrm{MgCl}_{2}$ on impaired transmembrane potential and protein synthesis in liver ischemia. Acta Chir. Scand. 148, 601-607.

10. Williamson, D.H., Mellanby, J. \& Krebs, H.A. (1962) Enzymatic determination of $\mathrm{D}(-)-\beta$-hydroxybutyric acid and acetoacetic acid in blood. Biochem. J. 82, 90-96.
11. Pearse, A.G. (1961) Histochemistry: Theoretical and Applied, pp. 167-178, Churchill J. Ltd., London.

12. Estabrook, R. \& Werringloer, J. (1978) The measurement of difference spectra: Application to the cytochromes of microsomes. Methods Enzymol. 52, 212-220.

13. Hodges, T. \& Leonard, R. (1974) Purification of a plasma membrane bound adenosine triphosphatase from plant roots. Methods Enzymol. 32, 392-406.

14. Shimizu, S., Kamiike, W., Hatanaka, N., Nishimura, M., Miyata, M., Inoue, T., Yoshida, Y., Tagawa, K. \& Matsuda, H. (1994) Enzyme release from mitochondria during reoxygenation of rat liver. Transplantation 57, 144148 .

15. Gonzales-Flecha, B., Cutrin, J.C. \& Boveris, A. (1993) Time course and mechanism of oxidative stress and tissue damage in rat liver subjected to in vivo ischemia-reperfusion. J. Clin. Invest. 91, 456-464.

16. Lemasters, J.J., Stemkowski, C.J., Ji, S. \& Thurman, R.G. (1983) Cell surface changes and enzyme release during hypoxia and reoxygenation in the isolated, perfused rat liver. $J$. Cell. Biol. 97, 778-786.

17. Settaf, A., Morin, D., Lamchouri, F., Elimadi, A., Cherrah, Y. \& Tillement, J.P. (1999) Trimetazidine ameliorates the hepatic injury associated with ischaemia-reperfusion in rats. Pharmacol. Res. 39, 211-216.

18. Izuishi, K., Hamamoto, I., Ichikawa, Y., Okano, K., Akram, H.M., Maeba, T. \& Tanaka, S. (1994) Effect of liver warm ischemia on the hepatic microsomal cytochrome P-450 monooxygenase system. Transplant. Proc. 26, 2417-2419.

19. Chien, K. \& Farber, J. (1980) Microsomal membrane dysfunction in ischemic liver cell. Arch. Biochem. Biophys. 77, 191-198. 